ARTIFICIAL SATELLITES, Vol. 52, No. 2 - 2017
DOI: 10.1515arsa-2017-0004

\title{
GNSS ANTENNA CAUSED NEAR-FIELD INTERFERENCE EFFECT IN PRECISE POINT POSITIONING RESULTS
}

\author{
Karol Dawidowicz, Radosław Baryła \\ University of Warmia and Mazury in Olsztyn, Institute of Geodesy, 10-719 Olsztyn, ul. \\ Oczapowskiego 1 \\ e-mail: karol.dawidowicz@uwm.edu.pl, radoslaw.baryla@uwm.edu.pl
}

\begin{abstract}
Results of long-term static GNSS observation processing adjustment prove that the often assumed "averaging multipath effect due to extended observation periods" does not actually apply. It is instead visible a bias that falsifies the coordinate estimation. The comparisons between the height difference measured with a geometrical precise leveling and the height difference provided by GNSS clearly verify the impact of the near-field multipath effect.

The aim of this paper is analysis the near-field interference effect with respect to the coordinate domain. We demonstrate that the way of antennas mounting during observation campaign (distance from nearest antennas) can cause visible changes in pseudo-kinematic precise point positioning results. GNSS measured height differences comparison revealed that bias of up to $3 \mathrm{~mm}$ can be noticed in Up component when some object (additional GNSS antenna) was placed in radiating near-field region of measuring antenna. Additionally, for both processing scenario (GPS and GPS/GLONASS) the scattering of results clearly increased when additional antenna crosses radiating near-field region of measuring antenna. It is especially true for big choke ring antennas. In short session $(15,30 \mathrm{~min}$.) the standard deviation was about twice bigger in comparison to scenario without additional antenna. When we used typical surveying antennas (short near-field region radius) the effect is almost invisible. In this case it can be observed the standard deviation increase of about $20 \%$. On the other hand we found that surveying antennas are generally characterized by lower accuracy than choke ring antennas. The standard deviation obtained on point with this type of antenna was bigger in all processing scenarios (in comparison to standard deviation obtained on point with choke ring antenna).
\end{abstract}

Keywords: near-field interference effects, GPS, GLONASS, PPP

\section{INTRODUCTION}

Generally we can distinguish two main station dependent errors which are of great concern for precise GNSS positioning applications: the phase center variations (PCV) of the receiving antenna and multipath (MP). Since both PCV and MP are different and independent errors their separation is a pre-requisite for their precise description and modeling. If we assume that there exist static and time-variant errors on a station the multipath can be considered as a time dependent error source. On the other hand for the phase center variations we can assume static behavior if we ignore factors like temperature dependencies and ageing. 
The site multipath influence itself can be separated into near-field and far-field effects (Balanis, 2005), which do have different properties. Near-field interference effects cause a systematic bias especially in the coordinate height component. Far-field interference effects theoretically may be averaged out by sufficient length of observation data.

The near-field multipath is increasingly of interest and importance in GNSS applications (Dilßner et al., 2008; Granström and Johansson, 2007; Wübbena et al., 2006; Wübbena et al., 2010). The GNSS signals are affected due to signal diffraction and reflection from objects in the close vicinity of the antenna and also by effects like imaging and electromagnetic interaction. Additionally the antenna near-field interference effect depends on antenna type, mount/setup, site, weather condition (Wübbena at al., 2010).

Although during the last decades the wide range of methods for multipath estimation and mitigation were developed (e.g. Bhuiyan and Lohan, 2010; Bilich and Larson, 2007; Christopher and Penina, 1998; LinLin et al., 2000; Rougerie et al., 2012; Satirapod et al., 2003; Yedukondalu et al., 2009) multipath signals still seriously affect the GNSS observables. In static applications with highest accuracy requirements, it is commonly assumed that multipath effects fully average out in long observation sessions. This assumption unfortunately is valid only in the case of short periodic multipath signals caused by distant objects located in the far-field region of the antenna (Volk and Levine, 1994). The signal reflections from the closest vicinity of the antenna introduces long periodic errors which are non-zero mean distributed and therefore introduce an unmodeled bias in the estimated parameters. This is especially true for setting and rising satellite. Additionally, reflecting objects located in the near-field region of the antenna can change also overall electromagnetic properties of the antenna (Dilßner et al., 2008).

There are also another reasons why multipath effects coming from the close vicinity can cause bigger problems compared to multipath from reflectors which are located further away from the antenna. Due to the short distance between the reflector and the antenna phase center, the reflected signals tend to be much stronger than signals coming from more distant objects (Wübbena at al., 2006).

The changing of the environment in the near-field around the antenna may change the reception characteristic and consequently affects the observed GNSS signals. As a result a systematic and complex error may affect the coordinates. Furthermore it was proven that there is an amplification of the near-field impact in the position domain (Wanninger and May, 2000). Although the range differences may have some millimeter, the coordinate bias can reach centimeters (Wanninger and May, 2000).

GNSS measurements are often carry with antennas mounted on massive concrete pillars or steel pylons. Since these structures are located in the near-field region, significant changes in the electromagnetic properties of the antennas can be expected. Additionally in many high precision surveying campaigns and monitoring tasks, different adaptation for the antenna mounts are designed, which can also influence electromagnetic properties of the antennas. Recently it appears also more and more measuring sets with antennas mounted very close to each other.

Research on improving GNSS measurement accuracy in difficult measurement conditions using three GNSS receivers are also carried out for several years (Bakuła, 2012; Hasegawa and Yoshimura, 2003; Valbuena et al., 2010). The survey unit consists of three GNSS receivers positioned on a special base. The central GNSS receiver is always positioned on a vertical line from the point being determined, and the other two receivers are set at a distance of $0.5 \mathrm{~m}$ from it. As a consequence, in each control point is a small subnetwork consisting of 
three receivers: left, middle, and right. So close fixed antennas can also be a source of mutual near-field multipath.

The problem of near-field multipath is also very important in GNSS antenna calibration issue. In (Wübbena, 2010) two typical geodetic setups with a tribrach on a quadratic and a round pillar reconstruction were selected to show up the influence of the near-field multipath on a Dorne Margoline choke ring antenna. The influence of the near-field interference effect had a magnitude of up to $7.5 \mathrm{~mm}$ in low elevations (for some regions even larger) and even 5 $\mathrm{mm}$ for $10 \mathrm{deg}$ elevation. This is probably the reason, why even for individually calibrated GNSS antennas height changes are observed for some sites, when the antenna is changed.

In (Lasparre, 2006) has been proven that the influence of the mounting mast on a non choke ring GNSS antenna phase centre can be almost a centimeter for the mean as well as for the elevation and azimuth dependant variation. This effect can be more than three times as large, due to different influence on the phase centre variations for the L1 and the L2 frequency. The resulting impact of the mounting mast can be more than $3 \mathrm{~cm}$ in the measured height. To resolve the problem it was proposed a procedure to calibration of the antenna including the upper part of the mast.

In (Dilßneret al., 2008) the objective was to give an insight into the near-field interference effect with respect to the coordinate domain. It was demonstrate that mechanical structures mounted underneath the antennas (e.g. tripods, tripod heads, etc.) can cause significant changes in the phase center offset and variations (PCV) described by the common spherical harmonic model during antenna calibration procedure. For the GPS carrier signals L1 and L2 these changes were in the order of several millimeters.

The aim of this paper is analysis the near-field interference effect with respect to the coordinate domain in pseudo-kinematic precise point positioning (PPP) results. PPP is a stand-alone positioning technique using undifferenced dual-frequency code and phase observations. Precise satellite orbit and clock, and accurate physical models are required to achieve highly accurate results. PPP provides a positioning solution in a dynamic, global reference frame, preventing any local distortions associated with differential positioning techniques. The use of a single GNSS receiver for PPP also has a number of disadvantages, the most significant being the long time span (about $20 \mathrm{~min}$ ) necessary for the ambiguity float solution to converge (Choy et al., 2016). Additionally, as no differencing techniques are used in PPP algorithms and since relative ionospheric measurement delays can't be assumed to cancel, dual-frequency receivers are required for high-precision positioning. Details on the achievements and limitations of PPP can be found, among others, in (Dawidowicz and Krzan, 2014; Kouba and Héroux, 2001; Krzan et al., 2016; Rizos et al., 2012; Zumberge et al. 1997). In this investigation we demonstrate that the way of antennas mounting during observation campaign (distance from nearest antennas) can cause visible changes in results. GNSS measured height differences comparison revealed that bias of up to $3 \mathrm{~mm}$ can be noticed in Up component when some object (additional GNSS antenna) was placed in near-field region of measuring antenna. Additionally for both processing scenario (GPS and GPS/GLONASS) the scattering of results clearly increase when additional antenna is mount in near-field radiating region of other antenna. It is especially true for big choke ring antennas. When we used typical surveying antennas (short near-field region radius) the effect is almost invisible. On the other hand we found that results obtained using surveying antennas are generally characterized by lower accuracy than results obtained using choke ring antennas. 


\section{RADIATING FIELD OF THE ANTENNA}

So far receiver based multipath mitigation techniques generally focus on C/A and L2C codeobservables. Unfortunately these techniques are ineffective in the case of a short excess signal path $\Delta \mathrm{d}$ (Bhuiyan and Lohan, 2010; Rougerie et al., 2012).

If the direct and the indirect signals arrive e.g. within $100 \mathrm{~ns}$, the modern receiver processing algorithms cannot distinguish between these two signals. For carrier phase multipath, it is assumed that the boundary where mitigation is impossible occur approximately at $\Delta d_{\min }=1 \mathrm{~m}$ (Weill, 1997).

The geometric multipath model described previously is only a useful approximation for the multipath error, it does not describe the electromagnetic field arising in the immediate vicinity of the antenna. Balanis (2005) subdivide the radiating field of an antenna into three regions: reactive near-field, radiating near-field and far-field (Fig. 1).

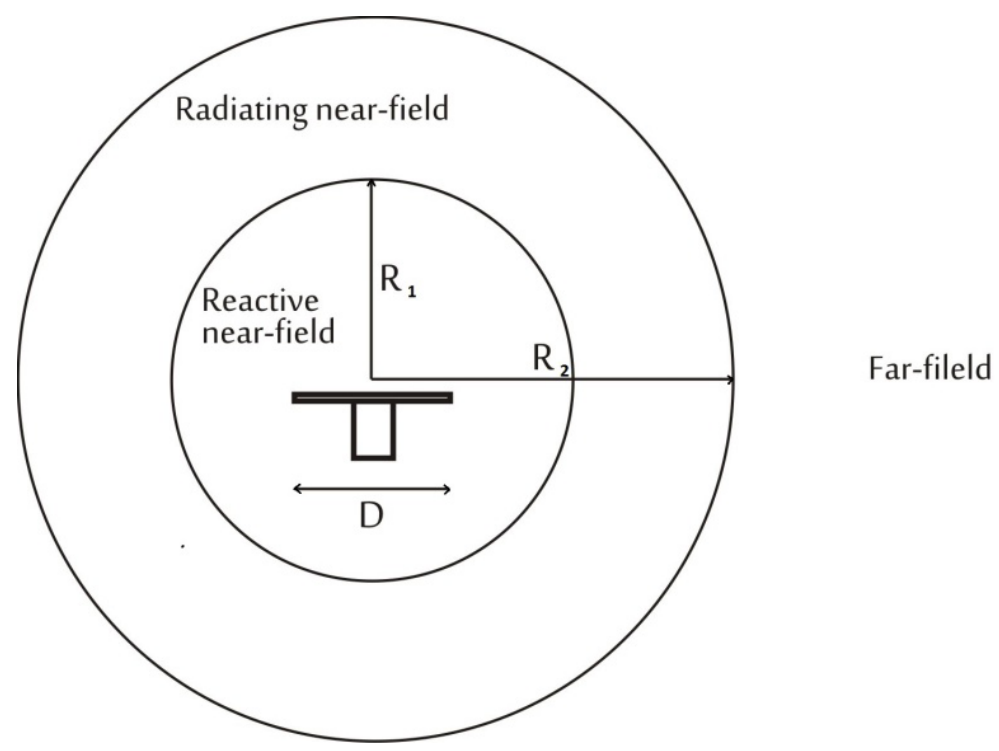

Fig. 1. Near- and far-fields of the antenna

(source: own work based on Dilßner et al. (2008))

The boundaries separating these regions depend on the wavelength $\lambda$ of the signal and the antenna diameter D. For GNSS antennas operating in the ultra high frequency range, the farfield region is generally taken to begin at a distance of:

$$
R_{2}=\frac{2 D^{2}}{\lambda}
$$

In the far-field region, geometrical ray optics is an appropriate method to describe the wave propagation. The wave front can be considered planar and rays are parallel. The shape of the radiation pattern is independent of the distance. Furthermore the electromagnetic characteristic of the antenna itself are not affected by objects located in the far-field region.

The distances where the simplified approximation breaks down are known as the near-field region. This region is subdivided into two more regions (Balanis, 2005). The conversion from the reactive near-field to the far-field is called radiating near-field region. The inner boundary of this region can be calculated according to formula:

$$
R_{1}=0.62 \cdot \sqrt{\frac{D^{3}}{\lambda}}
$$

and the outer boundary is $\mathrm{R}_{2}$. Within the radiating near-field region the radiation pattern depends on the distance $r$ from the antenna. If the maximum antenna dimension $D$ is very small compared to $\lambda$ the region may not exist. 
The near-field region is of particular importance since it forms the far-field transmission or reception pattern of an antenna. It can be visualized as a resonant reservoir that stores energy in the air surrounding the antenna. If any materials with higher permittivity than air occur in the near-field region, energy can be absorbed and not be available for the antenna. In the worst case, the occurrence of conductive structures changes the effective size of the antenna reception element and becomes part of the antenna (Dilßner et al., 2008).

Fig. 2 present simulated boundaries of radiating regions of an antenna according to formulas 1 and 2 calculated for two GPS frequency as well as using the so-called "ionosphere-free linear combination" - IF (double-frequency observation variant) and a different antenna diameters.
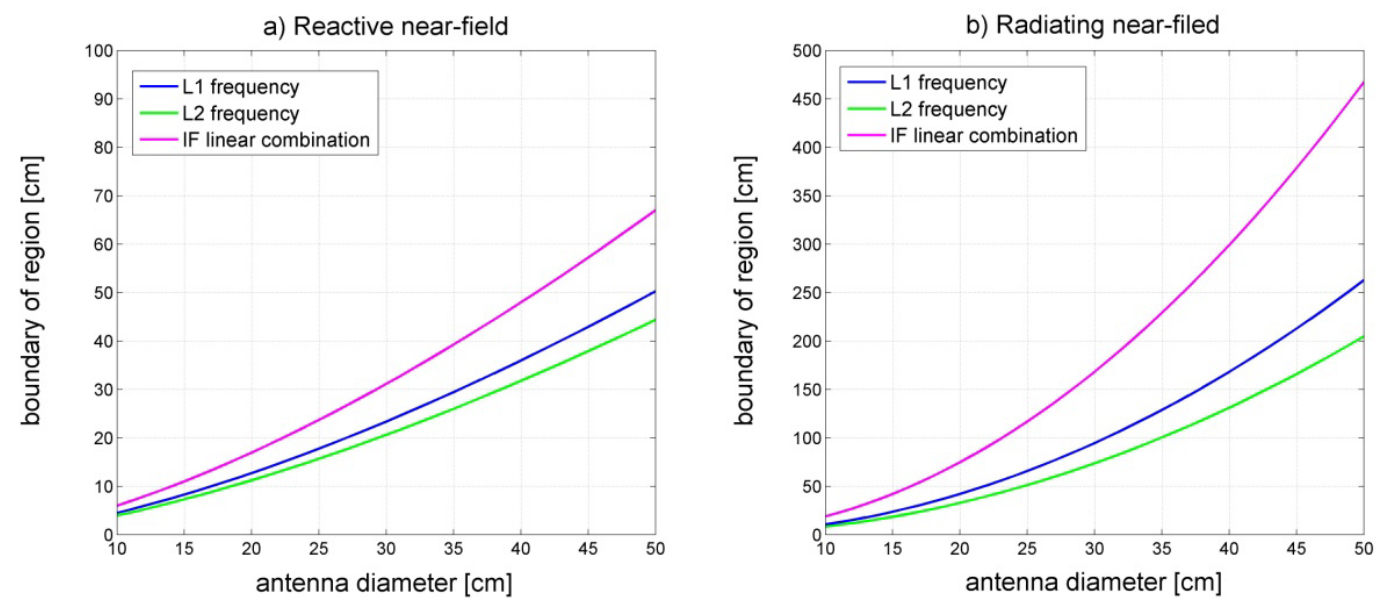

Fig. 2. Simulated boundaries of near-field regions of the antenna

The boundaries of radiating regions visible increases for large antennas (large antenna diameter) and in case of use double-frequency observation ("ionosphere-free linear combination" variant).

\section{RESEARCH STRATEGY}

Analyses were based on 6 days observation carried out in May 2015 using different antennas location (Table 1). In I and III measurement modes to mitigate the near-field interference effects in observations the antennas was installed at a suitable distance. Each test antenna candidate was mounted on the end of 1,60 $\mathrm{m}$ steel beam (pillar 1 and 3: PIL1 and PIL3) on the roof of the building in order to minimize the effect of near-field multipath in observations. In Mode II additional antenna was mounted on pillar 2 (PIL2). The following GNSS parameters were assumed for measurements: sampling interval $5 \mathrm{~s}$, minimum satellite elevation $0^{\circ}$. The zero minimum elevation was chosen for the purpose of analysis. As is well known signals coming from low elevation are more sensitive to the multipath effect. 
Table 1. Antennas and receivers configuration during test

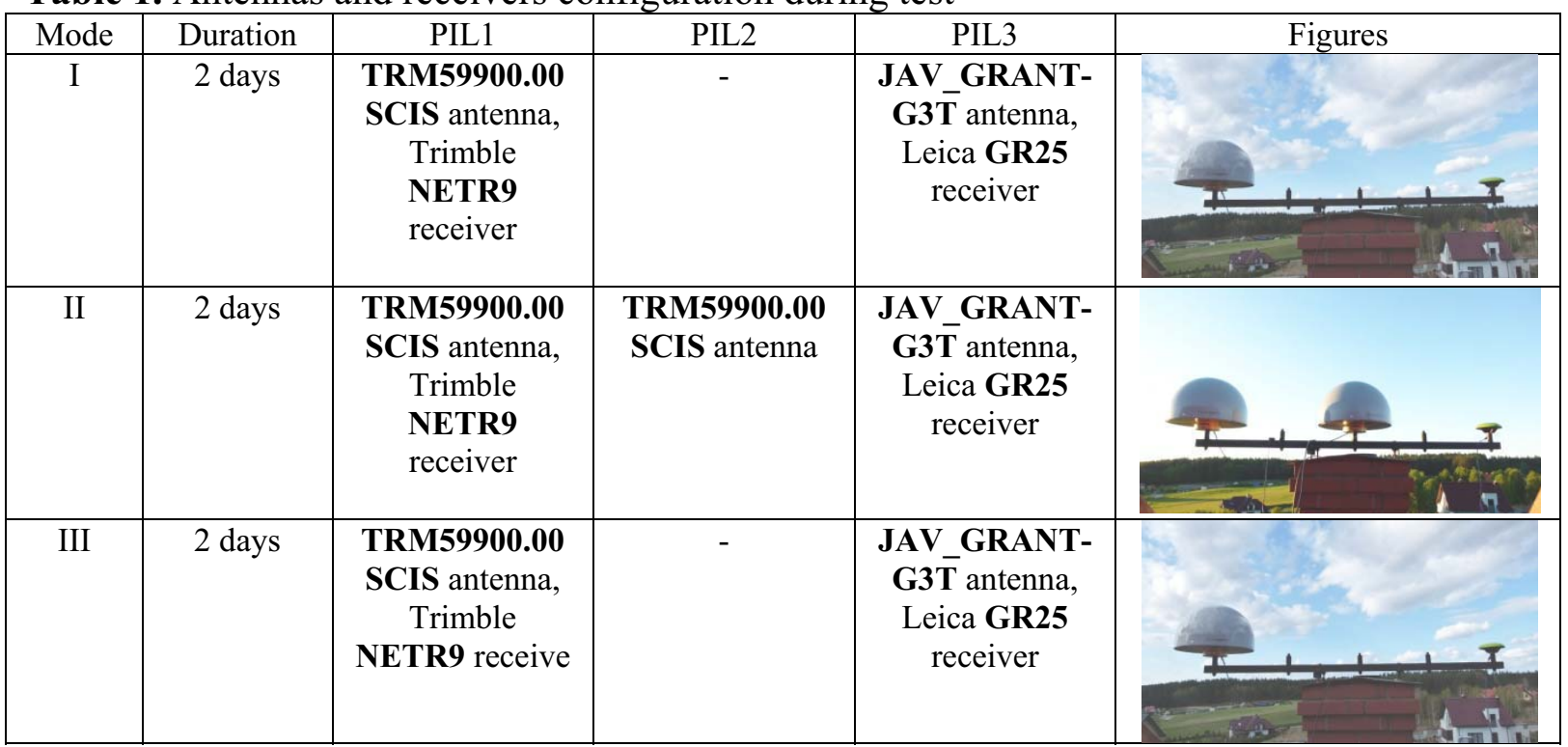

Each "mode" was processed separately using "ionosphere-free linear combination" - IF (dualfrequency observation variant). This combination allows to eliminate ionosphere refraction but, on the other hand the observational noise of the IF linear combination is larger by a factor of $\sim 3$ than that for L1 only observations and IF combinations also considerably amplify systematic effects due to multipath, antenna phase center offsets and variations, etc. (Schaer, 1999).

In computation standard processing strategy was used. Some options are presented below:

- ESA final orbits and clock products,

- ocean loading corrections applied (IERS 2010 convention),

- elevation dependent weighting function: $1 / \cos (\mathrm{z})$,

- troposphere model: Saastamoinen with Global Pressure and Temperature (GPT) model, Global Mapping Function (GMF) and estimated parameters,

- float carrier-phase ambiguities,

- processing frequency: IF formed from L1 and L2 frequencies.

Balanis (2005) subdivide the radiating field of an antenna into three regions: reactive nearfield, radiating near-field and far-field. Table 2 presents boundaries of multipath regions calculated for both test antennas (GPS and GLONASS system). Because for GLONASS the wavelengths of the carrier are different for each satellite due to the different frequencies used these calculation were based on L1 and L2 centre frequency. It should be noted that the wavelength difference for L1 frequency between the two extremes is $0.15 \mathrm{~cm}$, which is less than $0.01 \mathrm{~L} 1$ cycle. The antenna diameters were based on factory data.

Table 2. The boundaries of near-field regions for used in test antennas.

\begin{tabular}{|c|c|c|c|c|}
\hline \multirow{2}{*}{ Frequency } & \multicolumn{2}{|c|}{ TRM59900.00 SCIS } & \multicolumn{2}{c|}{ JAV_GRANT-G3T } \\
\cline { 2 - 5 } & \multicolumn{2}{|c|}{ Diameter D $=38 \mathrm{~cm}$} & \multicolumn{2}{c|}{ Diameter D=14 cm } \\
\hline GPS & R1 [cm] & R2 [cm] & R1 [cm] & R2 [cm] \\
\hline L1 & 33.3 & 151.8 & 7.4 & 20.6 \\
\hline L2 & 29.4 & 118.3 & 6.6 & 16.1 \\
\hline GLONASS (Centre) & R1 [cm] & R2 [cm] & R1 [cm] & R2 [cm] \\
\hline L1 & 33.1 & 150.4 & 7.4 & 20.4 \\
\hline L2 & 29.6 & 119.8 & 6.6 & 16.3 \\
\hline
\end{tabular}

According to the far-field condition, the radius of the sphere defining the boundary between near-field and far-field for JAV_GRANT-G3T antenna is $20.6 \mathrm{~cm}$ (L1 frequency). For the 
reference chock ring TRM59900.00 SCIS antenna equipped with the conventional extended ground plane the boundary is located at $151.8 \mathrm{~cm}$ (L1 frequency). Therefore, we can conclude that the both antennas are well located outside the near-field region. In setup II additional antenna were located in middle of steel beam in order to cross the boundaries of near-field multipath regions.

Analysis of the results was carried out in two steps. Firstly we compare North, East and Up residuals time series repeatability - we check if there is a structure in the residuals. Then we have a look on the residuals statistical behavior. The results of calculation and their discussion are provided in next section.

\section{ANALYSIS OF RESULTS}

24-hour observations were divided into 15, 30 and 60 minutes sessions and processed using PPP technique in GPS-only and GPS/GLONASS (GNSS) observation scenario. The processing was done using the NAPEOS ver. 3.3.1. Several PPP runs, leaving all processing options identical, except the session duration and used observation (GPS-only vs. GPS/GLONASS), were performed. Generally, 15 minute-long sessions may be too short for PPP. However the problem will be occurred if the use of a 15 minute window caused the PPP solution has not converged. Fortunately in the presented results, all solutions are converged. Figures 3-7 and Tables 3-5 present achieved results.

\subsection{Point coordinates analysis}

The position residuals are calculated from the differences between the 'true' coordinates with the estimated values. The average position from the four 24-h sessions (Mode I and Mode III - without antenna on PIL2 in order to minimize the effect of near-field multipath in results) was adopted as the 'true' position. Residuals of coordinate components time series obtained in 15-minutes session processing are presented on Figure 3 as box whisker plots. Some results (mean coordinate difference and standard deviation calculated from post-fit residuals) are also presented in Table 3.
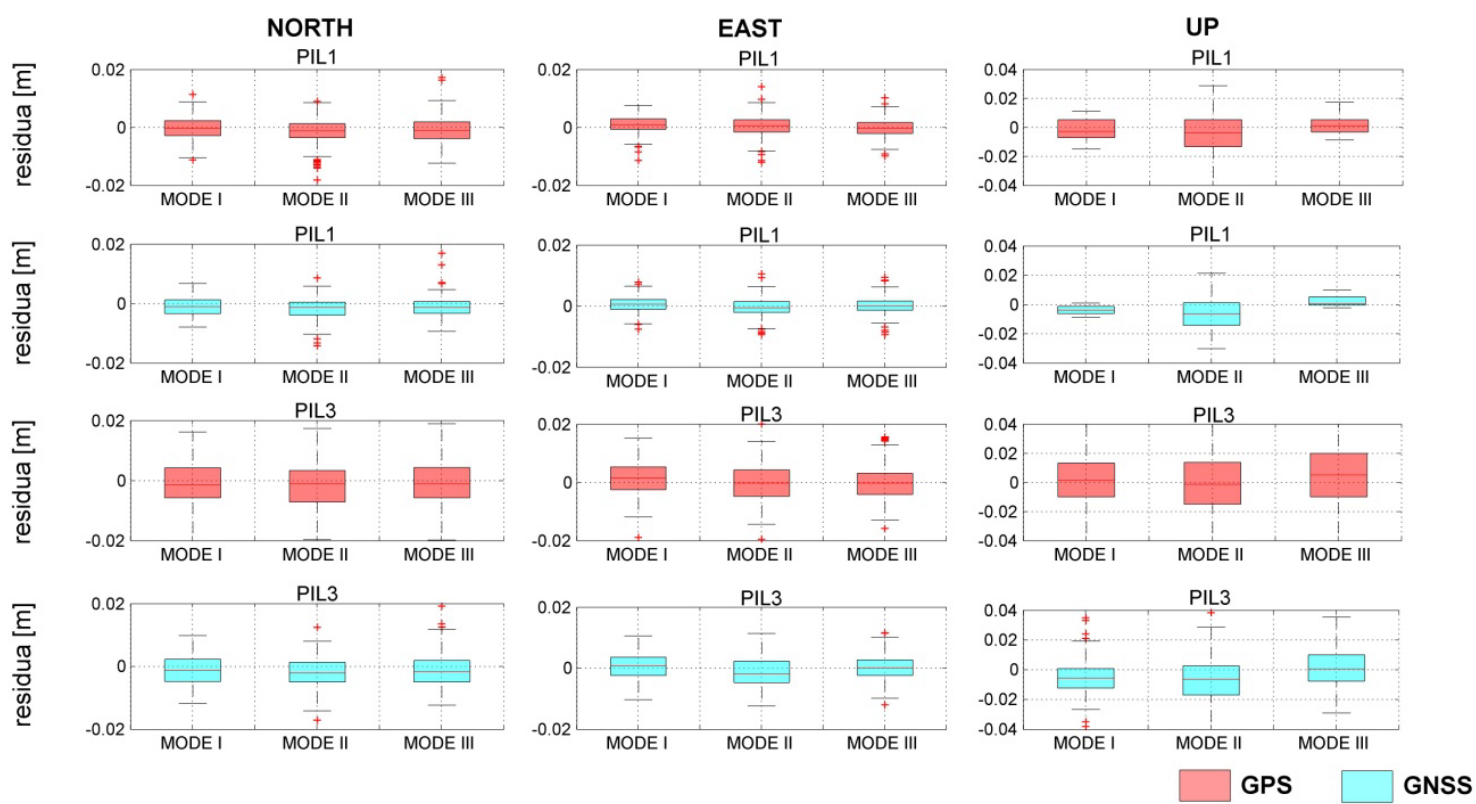

Fig. 3. Box whisker plots of the residuals obtained in 15-minutes session processing.

Results obtained in processing of 15-minutes session reveal the following:

- the presented residuals in North and East are visible smaller than in Up component, 
- residuals obtained in GPS-only processing are bigger than residuals obtained in GPS/GLONASS processing,

- residuals obtained on PIL3 (JAV_GRANT-G3T antenna) are bigger than residuals obtained on PIL1 (TRM59900.00 SCIS antenna),

- residuals obtained in Mode II (where additional TRM59900.00 SCIS antenna was mounted on PIL2) are bigger than residuals obtained in Mode I and Mode III (this is especially visible for Up component on PIL1),

- small mean coordinate differences were also observed when results obtained in three modes were compared.

Presented in Table 3 results confirm earlier remarks. North and East components have better accuracy than Up component. In some cases (e.g.: PIL1 (GPS) Mode II; PIL1 (GNSS) Mode II; PIL3 (GNSS) Mode I and II) standard deviation (SD) of Up component is three times bigger than SD obtained for horizontal coordinates. Processing combined GPS/GLONASS observations may also allow to obtain better accuracy than processing GPSonly observations. Comparison of SD obtained in GPS-only processing variants and corresponding SD obtained in GNSS (GPS+GLONASS) processing reveals, in some cases (e.g.: PIL3, Mode I, North coordinate), its reduction of nearly half. We found also that using survey-grade antennas may result in obtaining a lower accuracy (about two times bigger SD) in comparison to results obtained using choke ring antennas. Finally we proved that placement another antenna in radiating near-field of measuring antenna may cause effect which is clearly visible in results by lowering accuracy, especially in Up component (PIL1). SD obtained in Mode II is visible larger in comparison to SD obtained in Modes I and III. Analyzing mean coordinate differences, obtained from three test modes, some discrepancies can be observed (e.g.: PIL1 (GNSS) Up coordinate: $9 \mathrm{~mm}$ ). Unfortunately it is difficult to find any systematic behavior of these differences.

Table 3. Statistical characteristics for 15 -minutes session processing results.

\begin{tabular}{|c|c|c|c|c|c|c|}
\hline \multirow{2}{*}{$\begin{array}{c}\text { POSITION } \\
\text { COMPONENT }\end{array}$} & \multicolumn{3}{|c|}{ MEAN DIFFERENCE [mm] } & \multicolumn{3}{|c|}{ STANDARD DEVIATION [mm] } \\
\hline & MODE I & MODE II & MODE III & MODE I & MODE II & MODE III \\
\hline & \multicolumn{6}{|c|}{ PIL1 (GPS) } \\
\hline NORTH & -0.5 & -1.8 & -1.0 & 4.0 & 4.8 & 4.6 \\
\hline EAST & 0.9 & 0.3 & -0.3 & 3.0 & 3.8 & 3.2 \\
\hline \multirow[t]{2}{*}{ UP } & -1.4 & -4.9 & 1.9 & 7.5 & 14.2 & 6.6 \\
\hline & \multicolumn{6}{|c|}{ PIL3 (GPS) } \\
\hline NORTH & -0.8 & -1.8 & -0.6 & 8.3 & 8.5 & 8.3 \\
\hline EAST & 1.2 & -0.2 & -0.2 & 5.7 & 6.8 & 5.9 \\
\hline \multirow[t]{2}{*}{ UP } & 1.8 & 0.9 & 5.0 & 18.1 & 22.5 & 20.7 \\
\hline & \multicolumn{6}{|c|}{ PIL1 (GNSS) } \\
\hline NORTH & -1.1 & -1.7 & -1.3 & 3.1 & 3.6 & 3.6 \\
\hline EAST & 0.6 & -0.4 & 0.1 & 2.5 & 3.2 & 2.8 \\
\hline \multirow[t]{2}{*}{ UP } & -3.9 & -7.0 & 2.2 & 2.8 & 10.5 & 3.6 \\
\hline & \multicolumn{6}{|c|}{ PIL3 (GNSS) } \\
\hline NORTH & -1.4 & -2.1 & -1.3 & 4.6 & 5.2 & 5.3 \\
\hline EAST & 0.7 & -1.5 & 0.1 & 4.2 & 4.8 & 4.1 \\
\hline UP & -5.5 & -5.9 & 1.1 & 11.4 & 15.7 & 13.1 \\
\hline
\end{tabular}

Residuals of coordinate components time series obtained in 30-minutes session processing are presented below (Figure 4 and Table 4). 

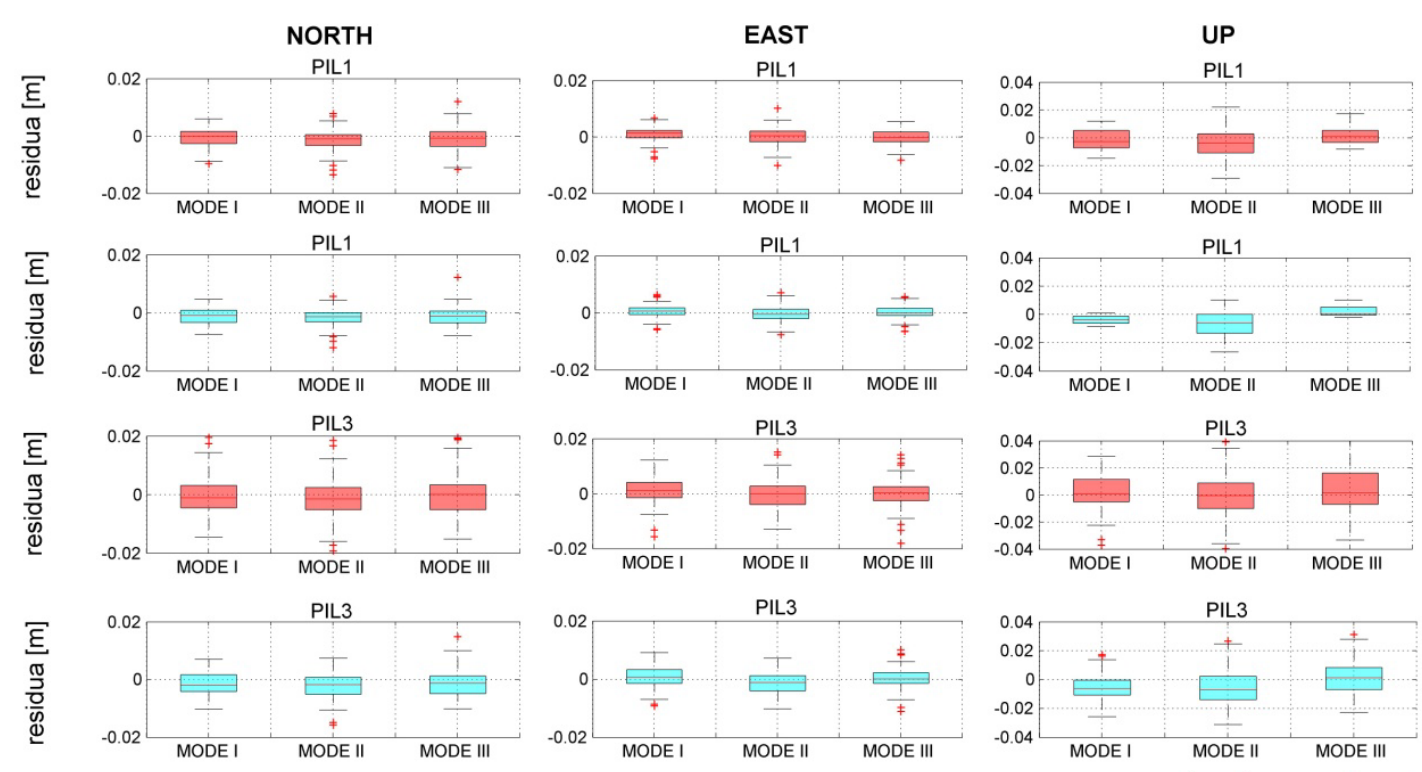

GPS

GNSS

Fig. 4. Box whisker plots of the residuals obtained in 30-minutes session processing.

As can be expected the scattering of residuals obtained in 30-minutes session show a reduction in comparison to 15 -minutes session. SD, on average, is about $20 \%$ smaller in this processing variant in comparison to 15 -min. processing results. In general, obtained in 30min. session processing results confirm all previous findings: - North and East components have better accuracy than Up component; - processing combined GPS/GLONASS observations may also allow to obtain better accuracy than processing GPS-only observations; - using survey-grade antennas may result in obtaining a lower accuracy in comparison to results obtained using choke ring antennas; - placement another antenna in radiating near-field of measuring antenna may cause effect which is clearly visible in results by lowering accuracy.

Table 4. Statistical characteristics for 30-minutes session processing results.

\begin{tabular}{|c|c|c|c|c|c|c|}
\hline \multirow{2}{*}{$\begin{array}{c}\text { POSITION } \\
\text { COMPONENT }\end{array}$} & \multicolumn{3}{|c|}{ MEAN DIFFERENCE [mm] } & \multicolumn{3}{|c|}{ STANDARD DEVIATION [mm] } \\
\hline & MODE I & MODE II & MODE III & MODE I & MODE II & MODE III \\
\hline & \multicolumn{6}{|c|}{ PIL1 (GPS) } \\
\hline NORTH & -0.6 & -1.6 & -1.1 & 3.6 & 3.9 & 4.0 \\
\hline EAST & 1.0 & 0.2 & -0.2 & 2.5 & 3.1 & 2.6 \\
\hline \multirow[t]{2}{*}{ UP } & -1.4 & -4.5 & 1.9 & 7.6 & 10.0 & 6.6 \\
\hline & \multicolumn{6}{|c|}{ PIL3 (GPS) } \\
\hline NORTH & -0.6 & -2.0 & -0.6 & 7.1 & 7.6 & 7.3 \\
\hline EAST & 1.3 & -0.3 & 0.0 & 4.7 & 5.3 & 5.0 \\
\hline \multirow[t]{2}{*}{ UP } & 1.6 & 0.3 & 4.7 & 14.3 & 18.1 & 16.7 \\
\hline & \multicolumn{6}{|c|}{ PIL1 (GNSS) } \\
\hline NORTH & -1.2 & -1.7 & -1.3 & 2.9 & 2.9 & 3.1 \\
\hline EAST & 0.6 & -0.5 & 0.1 & 2.2 & 2.7 & 2.4 \\
\hline \multirow[t]{2}{*}{ UP } & -3.9 & -7.0 & 2.3 & 2.8 & 8.7 & 3.6 \\
\hline & \multicolumn{6}{|c|}{ PIL3 (GNSS) } \\
\hline NORTH & -1.5 & -2.2 & -1.3 & 3.9 & 4.4 & 4.6 \\
\hline EAST & 0.7 & -1.6 & 0.2 & 3.6 & 3.7 & 3.4 \\
\hline UP & -5.9 & -5.7 & 1.2 & 9.5 & 12.5 & 11.1 \\
\hline
\end{tabular}

Results obtained in 60-minutes session processing are presented on Figure 5. Further extension of session length led to improving the results. Again residuals became smaller in all three position components. It is especially visible for PIL3 (GNSS) results, where SD was 
reduced by about half in comparison to 15 -min. session results. As previously better results were obtained for combined GPS/GLONASS processing variant. The scattering of residuals obtained on PIL3 (survey-grade antenna) also in this variant are visible bigger than residuals obtained on PIL1 (scientific choke ring antenna).
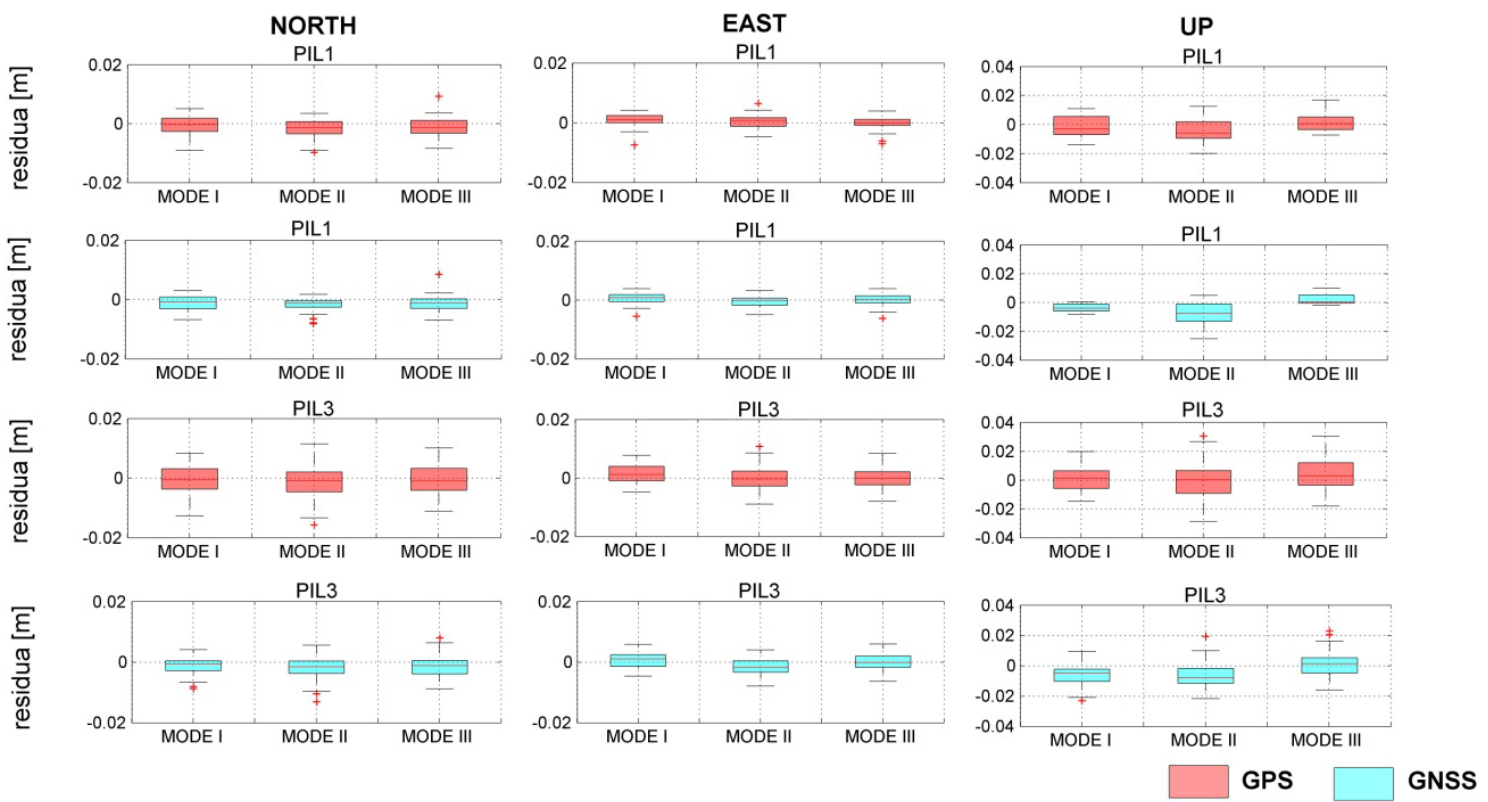

Fig. 5. Box whisker plots of the residuals obtained in 60-minutes session processing.

Table 5. Statistical characteristics for 60-minutes session processing results.

\begin{tabular}{|c|c|c|c|c|c|c|}
\hline \multirow{2}{*}{$\begin{array}{c}\text { POSITION } \\
\text { COMPONENT }\end{array}$} & \multicolumn{3}{|c|}{ MEAN DIFFERENCE $[\mathrm{cm}]$} & \multicolumn{3}{|c|}{ STANDARD DEVIATION [cm] } \\
\hline & MODE I & MODE II & MODE III & MODE I & MODE II & MODE III \\
\hline & \multicolumn{6}{|c|}{ PIL1 (GPS) } \\
\hline NORTH & -0.7 & -1.6 & -1.4 & 3.1 & 3.2 & 3.5 \\
\hline EAST & 0.9 & 0.2 & -0.2 & 2.0 & 2.4 & 2.1 \\
\hline \multirow[t]{2}{*}{ UP } & -1.4 & -5.2 & 1.8 & 7.5 & 8.7 & 6.6 \\
\hline & \multicolumn{6}{|c|}{ PIL3 (GPS) } \\
\hline NORTH & -0.3 & -1.6 & -0.4 & 4.7 & 5.3 & 5.1 \\
\hline EAST & 1.3 & -0.2 & -0.1 & 3.3 & 4.1 & 2.9 \\
\hline \multirow[t]{2}{*}{ UP } & 0.3 & -0.7 & 3.5 & 8.1 & 12.3 & 10.8 \\
\hline & \multicolumn{6}{|c|}{ PIL1 (GNSS) } \\
\hline NORTH & -1.1 & -1.6 & -1.4 & 2.5 & 2.3 & 2.6 \\
\hline EAST & 0.5 & -0.5 & 0.1 & 1.8 & 1.9 & 2.0 \\
\hline \multirow[t]{2}{*}{ UP } & -3.9 & -8.2 & 2.2 & 2.8 & 8.3 & 3.6 \\
\hline & \multicolumn{6}{|c|}{ PIL3 (GNSS) } \\
\hline NORTH & -1.3 & -2.1 & -1.4 & 3.0 & 3.6 & 3.5 \\
\hline EAST & 0.6 & -1.5 & 0.2 & 2.8 & 2.8 & 2.5 \\
\hline UP & -6.0 & -6.3 & 0.9 & 6.4 & 8.7 & 8.8 \\
\hline
\end{tabular}

In analyzing Table 5 it is visible that all previously described phenomena, are also present in 60-minutes session results. First of all it is also visible that placement another antenna in radiating near-field of measuring antenna may cause effect which is visible in the result scattering (especially in Up component). Unfortunately, when comparing mean differences obtained in the three modes it is difficult to find any systematic bias. To study if near-field interference effect can falsifies coordinates estimation and introduces systematic bias further analysis are needed. These analysis are presented below. 


\subsection{Height differences analysis}

In order to give a further proof of the existence of near-field interference effects, GNSS measured height differences were compared with the results from a precise levelling. The levelled height differences can be regarded as references, thus it is possible to quantify the absolute GNSS-accuracy. The pillar heights have been measured by precise levelling. The height differences between neighboring pillars are measured twice by precise doublelevelling. The differences between both solutions are smaller than $0.1-0.2 \mathrm{~mm}$. To get a first impression about analyzed phenomena, the differences between the precise levelling and the GNSS measurements are visualized in Fig. 6.
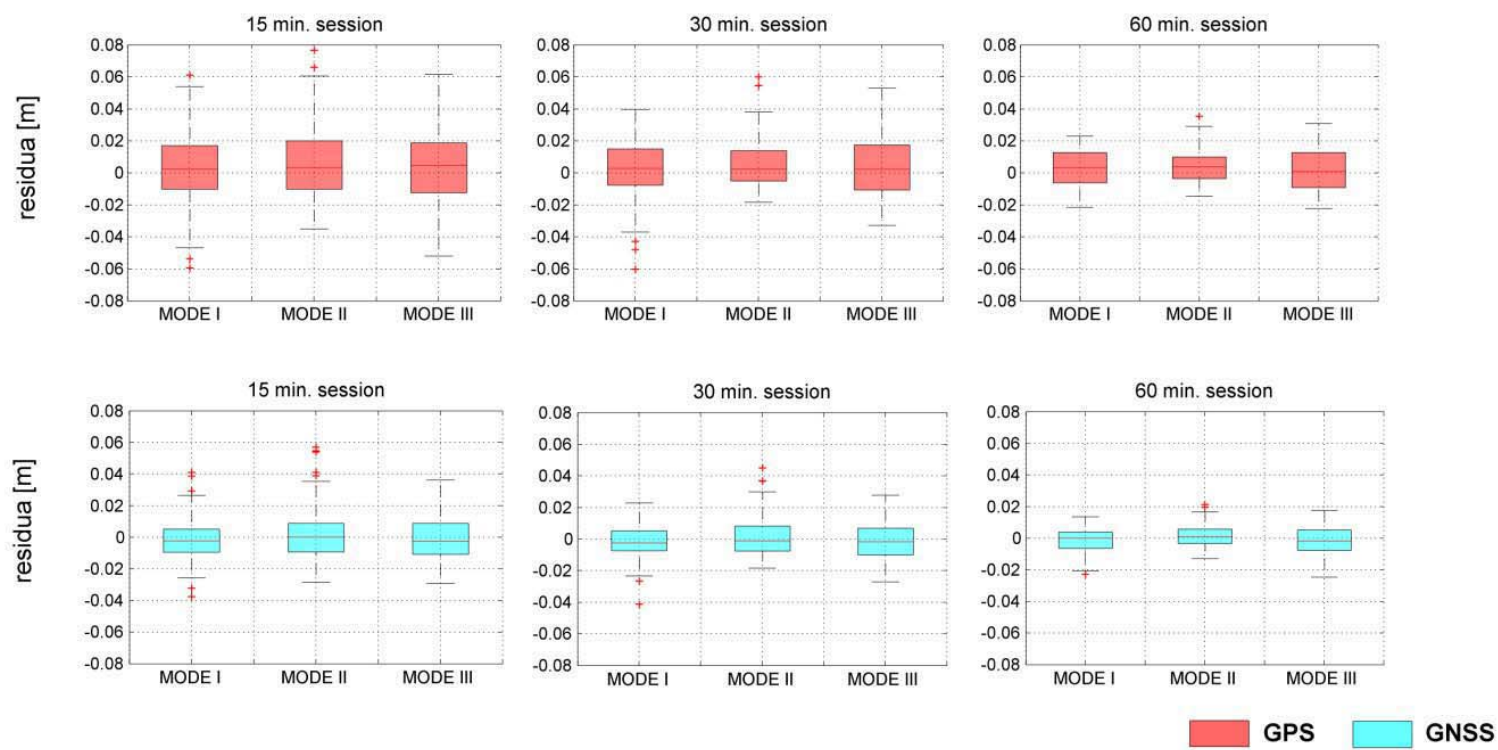

Fig. 6. Box whisker plots of the residuals obtained in height differences analysis.

Results obtained in height differences analysis reveal the following:

- residuals obtained in GPS-only processing are bigger than residuals obtained in GPS/GLONASS processing. This is true for all session duration variants.

- residuals get smaller with increasing time of measurement session,

- small systematic difference (bias) between mean height differences can be observed when we compare results obtained in MODE I/III and MODE II.

The table below (Table 6) shows mean coordinate difference and standard deviation (calculated from post-fit residuals) obtained in the height difference analysis.

Table 6. Statistical characteristics for height differences analysis.

\begin{tabular}{|c|c|c|c|c|c|c|}
\hline \multirow{2}{*}{$\begin{array}{c}\text { SESSION } \\
\text { DURATION }\end{array}$} & \multicolumn{7}{|c|}{ MEAN DIFFERENCE [cm] } & \multicolumn{3}{c|}{ STANDARD DEVIATION [cm] } \\
\cline { 2 - 7 } & MODE I & MODE II & MODE III & MODE I & MODE II & MODE III \\
\hline & 0.3 & 0.6 & 0.3 & 2.1 & 2.1 & 2.3 \\
\hline 15 min. & 0.3 & 0.5 & 0.3 & 1.8 & 1.5 & 1.9 \\
\hline 30 min. & 0.2 & 0.5 & 0.2 & 1.2 & 1.1 & 1.3 \\
\hline 60 min. & -0.2 & 0.1 & -0.1 & 1.2 & 1.6 & 1.3 \\
\hline & -0.2 & 0.1 & -0.1 & 1.1 & 1.2 & 1.2 \\
\hline 15 min. & -0.2 & 0.2 & -0.1 & 0.8 & 0.7 & 0.9 \\
\hline 30 min. & \multicolumn{7}{|c|}{ GNSS } &
\end{tabular}


Analyzing Table 6 significant differences can be seen in standard deviation values. In some case SD get twice smaller when we compare results obtained in 15-min. and 60-min. measurement session. Corresponding SD, obtained in GPS-only and GNSS variants, also differs. GPS-only solution generates almost two times bigger SD, in comparison to GNSS solution.

When we compare mean differences obtained in MODE I/III and MODE II systematic bias of up $3 \mathrm{~mm}$ can be noticed. This is obvious for all processing strategies (GPS/GNSS as well as 15, 30 and 60 minutes session). These comparisons clearly verify the impact of the near-field interference effect. In our experiment $3 \mathrm{~mm}$ bias in Up component differences occurred when some object (additional GNSS antenna) was placed in near-field region of measuring antenna. In our opinion this effect can be the result of near-field interference.

\section{CONCLUSIONS}

The aim of this paper is analysis the near-field interference effect with respect to the coordinate domain. We demonstrate that the way of antennas mounting during observation campaign (distance from nearest antennas) can cause visible changes in pseudo-kinematic precise point positioning results. GNSS measured height differences comparison revealed that systematic bias of up to $3 \mathrm{~mm}$ can be noticed in Up component when some object (additional GNSS antenna) was placed in near-field region of measuring antenna. These comparisons clearly verify the impact of the near-field interference effect. In our experiment $3 \mathrm{~mm}$ systematic bias in Up component differences occurred when some object (additional GNSS antenna) was placed in near-field region of measuring antenna. In our opinion this effect can be the result of near-field interference.

Additionally for both processing scenario (GPS and GPS/GLONASS) the scattering of results clearly increased when additional antenna was mount in near-field radiating region of measuring antenna. It is especially true for big choke ring antennas. In short session $(15,30$ min.) the standard deviation was about twice bigger in comparison to scenario without additional antenna. When we used typical surveying antennas (short near-field region radius) the effect is almost invisible. In this case it can be observed the standard deviation increase of about $20 \%$.

The results also indicate, that the choke-ring antenna is more accurate compared to the survey-grade antenna. The standard deviation obtained on point with this type of antenna was bigger in all processing scenarios (in comparison to standard deviation obtained on point with choke ring antenna). For permanently operating reference stations it is therefore an advantage to use a choke-ring antenna.

Acknowledgements. The authors are grateful for the possibility to use in field experiment TRM59900.00 SCIS antenna, Trimble NetR9 receiver and Leica GR25 receiver provided by the Head Office of Land Surveying and Cartography in Poland (GUGiK), the Geotronics Poland and the Leica Geosystems Poland, respectively.

\section{REFERENCES}

Bakuła M. (2012). An Approach to Reliable Rapid Static GNSS Surveying, Survey Review, 44(327), 265-271.

Balanis C. A. (2005). Antenna Theory. Third Edition. John Wiley \& Sons, New York.

Bilich A., Larson K. M. (2007). Mapping the GPS multipath environment using the signal-tonoise ratio (SNR), Radio Sci., Vol. 42, RS6003, DOI: 10.1029/2007RS003652. 
Bhuiyan M. Z. H., Lohan E. S. (2010). Advanced Multipath Mitigation Techniques for Satellite-Based Positioning Applications, International Journal of Navigation and Observation, ID 412393, DOI:10.1155/2010/412393.

Choy S., Bisnath S., Rizos Ch. (2016) Uncovering Common Misconceptions in GNSS Precise Point Positioning and its Future Prospect, International Federation of Surveyors, Article of the Month - September 2016.

Christopher J. C. Penina, A. (1998). Adaptive SNR-based carrier phase multipath mitigation technique, IEEE Transactions on Aerospace and Electronic Systems , 34(1), 264-276, January 1998.

Dawidowicz K. (2014). Analyzing the impact of different PCV calibration models on height determination using GPS/GLONASS observations from ASG-EUPOS network, Artificial Satellites, 49(4), 211-223.

Dilßner F., Seeber G. Wübbena G., Schmitz M. (2008). Impact of Near-Field Effects on the GNSS Position Solution, Proceedings ION GNSS-08, Savannah, Georgia.

Georgiadou Y., Kleusberg A. (1988). On carrier Signae signal multipath effects in relative GPS positioning, Manuscripta Geodaetica, 13, 172-179.

Granström C., Johansson J. (2007). Site Dependent Effects in High-Accuracy Applications of GNSS. Proceedings of the Symposium of the IAG Subcommission for Europe (EUREF), June 6-8, London.

Hasegawa, H., Yoshimura, T. (2003) Application of dual-frequecy GPS receiver for static surveying under tree canopy. Journal of Forest Research, Springer-Verlag, Tokyo Inc, $8(2), 103-110$.

Kouba, J., Héroux, P. (2001). Precise Point Positioning using IGS orbit and clock products, GPS Solutions, 5(2), 12-28, DOI: 10.1007/PL00012883.

Krzan G., Dawidowicz K., Stępniak K., Świątek K. (2016). Determining normal heights with the use of Precise Point Positioning, Survey Review, on-line version available et: http://www.tandfonline.com/doi/full, DOI: 10.1080/00396265.2016.1164939.

Lesparre J. (2006). The impact of the antenna mounting on the phase centre variation. EUREF Symposium 2006, on-line version available et: http://www.euref.eu/symposia/2006Riga/05-03.pdf.

LinLin G. E., Han S., Rizos C. (2000). Multipath mitigation of continuous GPS measurements using an adaptive filter, GPS Solutions , 4( 2), 19-30.

Rizos C., Janssen V., Roberts C., Grinter T. (2012). Precise Point Positioning: Is the era of differential GNSS positioning drawing to an end?, In: FIG Working Week 2012, Rome, Italy.

Rougerie S., Carrié G., Vincent F., Ries 1., Monnerat M. (2012). A New Multipath Mitigation Method for GNSS Receivers Based on an Antenna Array, International Journal of Navigation and Observation, ID 804732, DOI:10.1155/2012/804732.

Satirapod C., Khoonphool R., Rizos C. (2003). Multipath mitigation of permanent GPS stations using wavelets, International Symposium on GPS/GNSS, Tokyo, 2003.

Schaer S. (1999). Mapping and Predicting the Earth's Ionosphere Using the Global Positioning System. Ph.D. dissertation., Astronomical Institute University of Bern, Bern 1999, 205 pages. 
Yedukondalu, K., Sarma A. D., Satya Srinivas V. (2009). Multipath mitigation using LMS adaptive filtering for GPS applications, Applied Electromagnetics Conference (AEMC2009), Kolkata, India, December 14-16, 2009.

Valbuena R., Mauro F., Rodríguez-Solano R., Manzanera J.A. (2010). Accuracy and precision of GPS receivers under forest canopies in a mountainous environment, S. J. Agr. Res. 8(4), 1047-1057.

Volk C. M., Levine J. (1994). Analytical estimation of carrier multipath bias on GPS position measurements, National Institute of standards and Technology Technical Notes, Note 1366, 68 pages.

Wanninger L., May. (2000). Carrier Phase Multipath Calibration of GPS Reference Stations, Proceedings of ION GPS 2000, Salt Lake City, UT.

Weill L. R. (1997). Conquering Multipath: The GPS Accuracy Battle, GPS World, April, 5966.

Wübbena G., Schmitz M., Boettcher G. (2006) Near-field Effects on GNSS Sites: Analysis using Absolute Robot Calibrations and Procedures to Determine Corrections, Proceedings of the IGS Workshop 2006: Perspectives and Visions for 2010 and beyond, May 8-12, ESOC, Darmstadt, Germany.

Wübbena G., Schmitz M., Matzke N. (2010). On GNSS in-situ station calibration of nearfield multipath, International Symposium on GNSS, Space-based and Ground-based Augmentation Systems and Applications, November 29-30, 2010, Brussels, Belgium.

Zumberge J. F., Heflin M. B., Jefferson D. C., Watkins M. M., Webb F. H. (1997). Precise Point Positioning for the efficient and robust analysis of GPS data from large networks, Journal of Geophysical Research, 102(B3), 5005-5017, DOI: 10.1029/96JB03860.

Received: 2017-04-19,

Reviewed: 2017-04-24 and 2017-06-14,

Accepted: 2017-06-16. 